\title{
DETERMINATION OF OPTIMUM CLASSIFICATION SYSTEM FOR HYPERSPECTRAL IMAGERY AND LIDAR DATA BASED ON BEES ALGORITHM
}

\author{
F. Samadzadegan a , H. Hasani ${ }^{\text {a, * }}$ \\ ${ }^{a}$ School of Surveying and Geospatial Engineering, College of Engineering, University of Tehran, Tehran, Iran - \\ (samadz, hasani)@ut.ac.ir
}

Commission I, ICWG III/VII

KEY WORDS: Hyperspectral, LiDAR, Fusion, SVM Classifier, Optimization, Parameter Determination, Feature Selection, Bees Algorithm

\begin{abstract}
:
Hyperspectral imagery is a rich source of spectral information and plays very important role in discrimination of similar land-cover classes. In the past, several efforts have been investigated for improvement of hyperspectral imagery classification. Recently the interest in the joint use of LiDAR data and hyperspectral imagery has been remarkably increased. Because LiDAR can provide structural information of scene while hyperspectral imagery provide spectral and spatial information. The complementary information of LiDAR and hyperspectral data may greatly improve the classification performance especially in the complex urban area. In this paper feature level fusion of hyperspectral and LiDAR data is proposed where spectral and structural features are extract from both dataset, then hybrid feature space is generated by feature stacking. Support Vector Machine (SVM) classifier is applied on hybrid feature space to classify the urban area. In order to optimize the classification performance, two issues should be considered: SVM parameters values determination and feature subset selection. Bees Algorithm (BA) is powerful meta-heuristic optimization algorithm which is applied to determine the optimum SVM parameters and select the optimum feature subset simultaneously. The obtained results show the proposed method can improve the classification accuracy in addition to reducing significantly the dimension of feature space.
\end{abstract}

\section{INTRODUCTION}

Recently, with the progress in remote sensing technologies, it is possible to measure different characteristics of objects on the earth such as spectral, height, amplitude and phase information by multispectral/hyperspectral, LiDAR and SAR respectively (Debes et al. 2014). Availability of different types of data, provides means of detecting and discriminating of land use land cover in complex urban area (Ramdani, 2013). Classification of urban area has been used in wide range of application, such as mapping and tracking, risk management, social and ecological problems (Fauvel, 2007).

Hyperspectral remote sensing data is characterized by a very high spectral resolution that usually results in hundreds of observation bands. According to spectral richness, it plays very important role in discrimination of land-cover with similar spectral reflectance (Chang, 2013). Although hyperspectral imagery provides comprehensive spectral information but classification of complex urban area based on just spectral information has some limitations: same objects with different spectral characteristic don't classify in a class (e.g. buildings with different roof material/color don't classify in one class) and different objects with same spectral appearance may classify in same class (e.g. tree and grass/ roof and road). On the other hand, LiDAR sensor provides 3D information from surfaces and mapping with LiDAR data depend on the ability to detect objects with different height. There is a complementary relationship between passive hyperspectral images and active LiDAR data, as they contain very different information (Khodadazadeh et al. 2015).

\footnotetext{
* Corresponding author
}

According to availability, robustness and accuracy of spectral and structural information of hyperspectral images and LiDAR data, fusion of hyperspectral images and LiDAR data in a joint classification system, may yield more reliable and accurate classification results. While there have been numerous investigations that have reported on the use of other multisensory data (e.g. LiDAR and Multispectral), very few results are available about simultaneously integration of these two data sources in classification tasks (Debes et al. 2014, Latifi et al. 2012).

This paper presents an optimum hybrid classification system by simultaneous determination of the SVM parameters and the selection of features through swarm optimization process in order to fuse hyperspectral imagery and LiDAR data.

\section{RELATED WORK}

During last years, some investigations were carried out on fusion/integration of hyperspectral images and LiDAR data in different application, such as forest structure analysis, urban area mapping, identification of tree species, forest fire management, etc. (Alonzo et al. 2014, Brook et al., 2010, Dalponte et al. 2008, Koetz et al. 2008, Latifi et al. 2012). In some research works, LiDAR data is used for separation of 2D and 3D objects and then hyperspectral images are applied to discriminate among different species of an object, such as roofing material (Niemann, et al., 2009; Zhang and Qiu, 2012). Sugumaran and Voss (2007), apply the object based classification where LiDAR data is used for segmentation and hyperspectral image to classify the segments.

Dalponte et al. (2008) merge a subset of hyperspectral bands with two LiDAR imaging data (intensity and nDSM), then fuse it with 
results of the image classified by SVM and Gaussian Mixture Model. Liu et al. (2011) compute Canopy Height Model (CHM) from first LiDAR return and Minimum Noise Fraction (MNF) transformation is executed based on the pixel-level fusion of hyperspectral imagery and CHM channels. Then the first 26 eigenvalue bands are kept as input data for SVM classifier. Latifi et al. (2012) fuse hyperspectral bands and LiDAR features using Genetic Algorithm (GA) and apply this to select the feature subset in order to model forest structure.

In order to optimize the classification performance of high dimensional data, several methods are proposed in literatures which can be categorized into three groups: parameter determination of classifier (Liu et al. 2014), feature selection (Rashedi and Nezamabadi-pour, 2014) and simultaneously consider both of them (Samadzadegan, et al. 2012). The parameters of classifier has significant effect on its performance where grid search is common way to determine them (Hsu et al. 2003). Moreover, the selection of the feature subset may affect several classification aspects, including classification accuracy, computation time, training sample size, and the cost associated with the features (Lin et al., 2008). Several studies are focused on optimization these two issues which show that according to the dependency of parameters and features, simultaneous parameter determination and feature selection yield the most accurate results (O'Boyle et al., 2008). Recently Liu et al. applied PSO to determine the SVM kernel and margin parameters in classification of hyperspectral imagery and the results compared with grid search method which show the superiority of the proposed method (Liu et al. 2014). Feature selection is another essential step in classification of high dimension data. Rashedi and Nezamabadi-pour (2014) proposed an improved version of the binary gravitational search algorithm as a tool to select the best subset of features with the goal of improving classification accuracy. As parameter values effect on feature subset selection and vice versa, Samadzadegan et al. (2012) show that the best performance of classification is obtained by simultaneously classifier determination and feature selection by Ant Colony Optimization.

In this paper an optimum hybrid classification system is presented that simultaneously determines SVM classifier parameters and selects the feature subset to optimize classification performance for combined hyperspectral imagery and LiDAR data.

\section{PROPOSED METHOD}

In order to fuse hyperspectral imagery and LiDAR data, a hybrid feature space consisting of spectral and structural features is generated. Spectral feature space composed of original hyperspectral bands, vegetation indices and principle components. On the other hand, textural analysis on normalized DSM (nDSM), roughness and its textures, slope descriptors are extracted from LiDAR data which make the structural feature space. By combining spectral and structural feature space, the hybrid feature space is defined. Then normalization is used to transform data into the range $[0,1]$, in order to reduce numerical complexity.

According to the stability of SVM in high dimensional space [5], SVM is selected as classifier. There are two important challenges in classification of high dimensional data by a SVM classifier: SVM parameter determination (kernel and regularization parameters) and feature subset selection. In order to optimize classification of this hybrid feature space based on SVM, optimized SVM parameters values and appropriate feature subsets should be selected. For this purpose the Binary Bees Algorithm, as a powerful population based optimization algorithm, is applied to determine SVM parameters and selection of features subset simultaneously. Figure 1 presents the flowchart of the proposed.

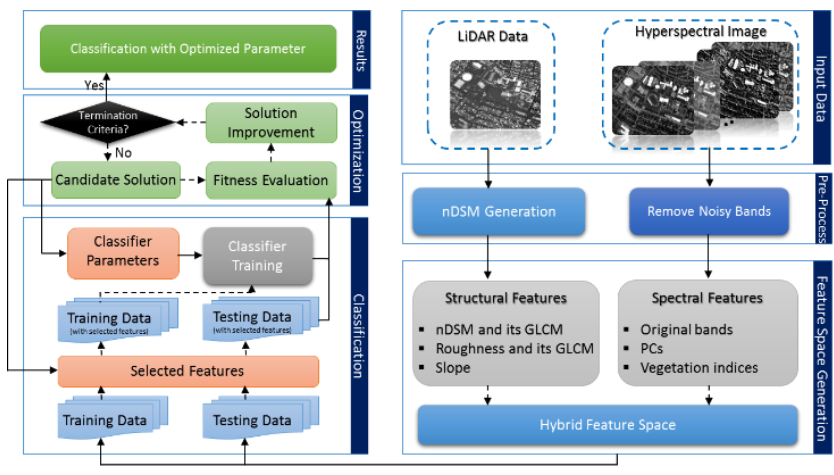

Figure 1. Flowchart of the proposed method

\subsection{Feature Space Generation}

Hyperspectral original bands include rich sources of spectral information but some indicators such as PCA components and vegetation indices may give additional information. Therefore PCA transformation is applied to the hyperspectral images and first three PCs are extracted additionally for use in the feature space. Then 30 vegetation indices are computed to discriminate vegetation classes from other classes (Table 1).

LiDAR-derived DSM provides height information, however more structural features should be generated to improve its ability in discrimination between classes. The nDSM is generated from DSM by geodesic morphological operation. In order to analyse the $\mathrm{nDSM}$ accurately, several types of features such as texture analysis, roughness and slope descriptors are extracted.

Grey Level Co-occurrence Matrices (GLCM) approach is used in this paper to extract second order statistical textural features from nDSM. In this paper, 16 features (Variance, Homogeneity, Contrast, Entropy, Dissimilarity, Sum Average, Angular Second Moment, Maximum Probability, Inverse Difference Moment, Sum Entropy, Sum Variance, Difference Variance, Correlation, Difference Entropy and two Information Measure of Correlation) are extracted from the GLCM matrix (Haralick et al. 1973).

Roughness is another structural feature which is extracted from $\mathrm{nDSM}$. For this purpose, the terrain roughness is parameterized by the standard deviation of the detrended $\mathrm{z}$-coordinates of the neighborhood. The plane is fitted to each neighborhood by the least square method and then the standard deviation of detrended height is determined. Texture analysis on the roughness map is also performed to better analysis of roughness. Moreover the slope of each neighbourhood in the nDSM is computed by applying the normal vector for the obtained plane which leads to a contribution of the slope feature to the structural feature space.

Finally, by stacking the spectral features from hyperspectral imagery and structural features from LiDAR data, the hybrid feature space is generated. 
Table 1. Spectral indices from hyperspectral image, $R_{x}$ is the reflectance at $x \mathrm{~nm}$

\begin{tabular}{|c|c|}
\hline Name & Equation \\
\hline Normalized Difference Vegetation Index (NDVI) & $\left(R_{800}-R_{670}\right) /\left(R_{800}+R_{670}\right)$ \\
\hline Simple Ratio (SR) & $R_{800} / R_{670}$ \\
\hline Enhanced Vegetation Index (EVI) & $2.5\left(\left(R_{800}-R_{670}\right) /\left(R_{800}+6 R_{670}-7.5 R_{475}+1\right)\right)$ \\
\hline Atmospherically Resistant Vegetation Index (ARVI) & $\left(R_{800}-2 R_{670}+R_{475}\right) /\left(R_{800}+2 R_{670}-R_{475}\right)$ \\
\hline Sum Green Index (SGI) & mean $\left(R_{i}\right), i=500, \ldots, 600$ \\
\hline Red Edge Normalized Difference Vegetation Index (RENDVI) & $\left(R_{750}-R_{705}\right) /\left(R_{750}+R_{705}\right)$ \\
\hline Modified Red Edge Simple Ratio Index (MRESRI) & $\left(R_{750}-R_{445}\right) /\left(R_{750}+R_{445}\right)$ \\
\hline Modified Red Edge Normalized Difference Vegetation Index (MRENDVI) & $\left(R_{750}-R_{705}\right) /\left(R_{750}+R_{705}-2 R_{445}\right)$ \\
\hline Vogelmann Red Edge Index 1, 2 (VREI 1) & $\left(R_{734}-R_{747}\right) /\left(R_{715}+R_{726}\right),\left(R_{734}-R_{747}\right) /\left(R_{715}+R_{720}\right)$ \\
\hline Red Edge Position Index (REPI) & wavelength of steepest slope within the range 690 to $740 \mathrm{~nm}$ \\
\hline Photochemical Reflectance Index (PRI) & $\left(R_{531}-R_{570}\right) /\left(R_{531}+R_{5700}\right)$ \\
\hline Structure Insensitive Pigment Index (SIPI) & $\left(R_{800}-R_{445}\right) /\left(R_{800}+R_{680}\right)$ \\
\hline Red Green Ratio Index (RGRI) & mean $($ red bands $) /$ mean $($ green bands $)$ \\
\hline Plant Senescence Reflectance Index (PSRI) & $\left(R_{680}-R_{500}\right) / R_{750}$ \\
\hline Carotenoid Reflectance Index 1, 2 (CRI 1,2) & $\left(1 / R_{510}\right)-\left(1 / R_{550}\right),\left(1 / R_{510}\right)-\left(1 / R_{700}\right)$ \\
\hline Anthocyanin Reflectance Index 1, 2 (ARI 1,2) & $\left(1 / R_{550}\right)-\left(1 / R_{700}\right), R_{800}\left[\left(1 / R_{550}\right)-\left(1 / R_{700}\right)\right]$ \\
\hline Modified Simple Ratio (MSR) & $\left(R_{800} / R_{670}-1\right) / \sqrt{R_{800} / R_{670}+1}$ \\
\hline Renormalized Difference Vegetation Index (RDVI) & $\left(R_{800}-R_{670}\right) / \sqrt{R_{800}+R_{670}}$ \\
\hline Soil Adjusted Vegetation Index (SAVI) & $(1.5)\left(R_{800}-R_{670}\right) /\left(R_{800}+R_{670}+0.5\right)$ \\
\hline Improved SAVI (MSAVI) & $1 / 2\left[2 R_{800}+1-\sqrt{\left(2 R_{800}+1\right)^{2}-8\left(R_{800}-R_{670}\right)}\right.$ \\
\hline $\begin{array}{l}\text { Modified Chrophyll Absorption Ration Index (MCARI) } \\
\text { MCARI1 }\end{array}$ & $\begin{array}{l}{\left[\left(R_{700}-R_{670}\right)-0.2\left(R_{700}-R_{550}\right)\right]\left(R_{700} / R_{670}\right)} \\
\quad 1.2\left[2.5\left(R_{800}-R_{670}\right)-1.3\left(R_{800}-R_{550}\right)\right] \\
\quad 1.5\left[2.5\left(R_{800}-R_{670}\right)-1.3\left(R_{800}-R_{550}\right)\right] \\
\end{array}$ \\
\hline MCARI2 & $\sqrt{\left(2 R_{800}+1\right)^{2}-\left(6 R_{800}-5 \sqrt{R_{670}}\right)-0.5}$ \\
\hline Triangular Vegetation Index (TVI) & $0.5\left[120\left(R_{750}-R_{550}\right)-200\left(R_{670}-R_{550}\right)\right]$ \\
\hline Modified TVI (MTVI) & $\begin{array}{l}1.2\left[1.2\left(R_{800}-R_{550}\right)-2.5\left(R_{670}-R_{550}\right)\right] \\
1.5\left[1.2\left(R_{800}-R_{550}\right)-2.5\left(R_{670}-R_{550}\right)\right]\end{array}$ \\
\hline MTVI2 & $\sqrt{\left(2 R_{800}+1\right)^{2}-\left(6 R_{800}-5 \sqrt{R_{670}}\right)-0.5}$ \\
\hline Water Band Index (WBI) & $R_{900} / R_{970}$ \\
\hline
\end{tabular}

\subsection{SVM Classifier}

SVM is a learning technique derived from statistical learning theory. It is calculating an optimally separating hyperplane that maximizes the margin between two classes. If samples are not separable in the original space, kernel functions are used to map data into a higher dimensional space with a linear decision function (Abe et al. 2010).

Given a dataset with $n$ samples $\left\{\left(x_{i}, y_{i}\right) \mid i=1, \ldots, n\right\}$ where $x_{i} \in \mathfrak{R}^{k}$ is a feature vector with $k$ components and $y_{i} \in\{-1,1\}$ denotes the label of $x_{i}$. The SVM looks for a hyperplane $w . \phi(x)+b=0$ in a high dimensional space, able to separate the data from classes 1 and -1 with a maximum margin. $w$ is a weight vector, orthogonal to the hyperplane, $\mathrm{b}$ is an offset term and $\phi$ is a mapping function which maps data into a high dimensional space to separate data linearity with a low training error. Maximizing the margin is equivalent to minimizing the norm of $w$. thus by solving the following minimization problem, SVM will be trained:

$$
\begin{gathered}
\text { Minimize: } \frac{1}{2}\|w\|^{2}+C \sum_{i=1}^{n} \xi_{i} \\
\text { Subject to: } y_{i}(w . \phi(x)+b) \geq 1-\xi_{i} \text { and } \xi_{i} \\
\geq 0, \text { for } i=1, \ldots, n
\end{gathered}
$$

where $C$ is a regularization parameter that imposes a trade-off between the number of misclassification in the training data and the maximization of the margin and $\xi_{i}$ are slack variables. The decision function obtained through the solution of the minimization problem in Equation (1) is given by:

$$
f(x)=\sum_{x_{i} \in S V} y_{i} \alpha_{i} \phi\left(x_{i}\right) \cdot \phi(x)+b
$$

where the constants $\alpha_{i}$ are called Lagrange multipliers determined in the minimization process. SV corresponds to the set of support vectors, training samples for which the associated Lagrange multipliers are larger than zero. The kernel functions compute dot products between any pair of samples in the feature space. Gaussian Radial Basic Function (RBF) is a common kernel which is used in this paper and it is defined by (3).

$$
K_{\text {Gaussian }}\left(x_{i}, x_{j}\right)=e^{\frac{-\left|x_{i}-x_{j}\right|}{2 \sigma^{2}}}
$$

In the proposed method, the classification module plays an important role in evaluation of the fitness function where SVM is trained by training data and trained SVM is evaluated by testing (unseen) data.

\subsection{Bees Algorithm Optimization}

Bees Algorithm is a meta-heuristic optimization algorithm that model the foraging behaviour of honey bee colony. The foraging process of honey bee colony in the nature begins in nature by scout bees which move randomly to search for promising flower patches. Flower patches with large amounts of nectar visited by more bees in neighbourhood of that site, whereas patches with less nectar receive fewer bees and other bee fly randomly for discovering new food source (Pham et al. 2006).

Bees Algorithm starts with the $n$ scout bee move randomly in the search space. The quality of the sites visited by the scout bees (each bee represents a candidate solution) are evaluated by fitness function. Then bees that have the highest fitnesses are selected and sites visited by them are chosen for neighborhood search $(m)$. In the next step, algorithm conducts searches in the neighborhood of the selected sites, assigning more bees to search near to the best $e$ sites. The bees are chosen directly according to 
the fitnesses associated with the sites they are visiting. Searches in the neighborhood of the best $e$ sites which represent more promising solutions are made more detailed by recruiting more bees to follow them than the other selected bees $(m-e)$. Together with scouting, this differential recruitment is a key operation of the Bees Algorithm.

However for each patch only the bee with the highest fitness will be selected to for the next bee population. In nature, there is no such a restriction. This restriction is introduced here to reduce the number of points to be explored. Then, the remaining bees in the population are assigned randomly around the search space scouting for new potential solutions. These steps are repeated until a stopping criterion is met (Pham et al. 2006).

\subsection{Determination of Optimum Classification System for Classification of Hyperspectral Imagery and LiDAR Based on Bees Algorithm}

In order to determine the SVM parameters values and feature subset simultaneously based on Bees Algorithm, binary coding is applied. In the proposed method, binary string composed of three main parts is considered: features, regularization parameter and kernel parameter. The first part of binary string consist of $n_{f}$ bits equal to dimension of feature space. Where ' 0 ' and ' 1 ' in the $i^{\text {th }}$ bit means that $i^{\text {th }}$ feature should be discard and considered, respectively. Regularization and kernel parameters are realvalued and transform to binary coding for consistency with the binary nature of the feature selection process. The length of regularization $\left(n_{c}\right)$ and kernel parameters $\left(n_{k}\right)$ depends on the range of the parameters and the required precision.

Evaluation of the candidate solution is done by using a fitness function. The first part of the binary of the solution define which feature should be selected. For the determination of the SVM parameters, the binary format of the second and third parts of the solution converts to a real-value, expressed by Equation (4).

$$
p=\min _{p}+\frac{\max _{p}-\min _{p}}{2^{l}-1} \times d
$$

where $p$ is the real value of the bit string, $\min _{p}$ and $\max _{p}$ are minimum and maximum values of the parameter $p$, determined by the user. $l$ is the length of the bit string (for each parameter) and $d$ is a decimal value of the bit string.

Results may have fewer selected features and a higher classification accuracy. The combination of classification accuracy and the number of selected features constitutes the evaluation function. Multiple criteria problems can be solved by creating a single objective fitness function that combines the two goals into one. The objective function is defined by Equation (5).

$$
f=\rho \times(1-\text { accuracy })+(1-\rho) \times \frac{1}{N_{f}}
$$

where $f$ is the fitness value, $\rho$ is a constant parameter in $[0,1]$, accuracy obtained by Kappa coefficient and $N_{f}$ is the number of selected features.

The proposed method starts with generation of the candidate solutions which are formed randomly at the first iteration. Then each bee (represent by candidate solution) is evaluated by Equation (5) and the bee with higher classification accuracy and the lowest selected feature subset is selected as promising solution for the population (with maximum fitness value). Neighbourhood search around the best solutions is performed by changing the value of a random bit of that solutions. Other bees are search randomly by generating the random binary string which show the candidate solutions. This process is iterated till the termination criterion (maximum iteration) is satisfied.

\section{EXPERIMENTAL RESULTS}

To evaluate the performance of the proposed method, experiments are performed on Compact Airborne Spectrographic Imager (CASI) hyperspectral imagery and LiDAR derived DSM acquired by the NSF-funded Center for Airborne Laser Mapping (NCALM), both at the same spatial resolution $(2.5 \mathrm{~m})$. The hyperspectral imagery consists of 144 spectral bands in the spectral range between $380 \mathrm{~nm}$ to $1050 \mathrm{~nm}$ and the corresponding co-registered DSM consists of elevation in meters above sea level (Geoid 2012A model).

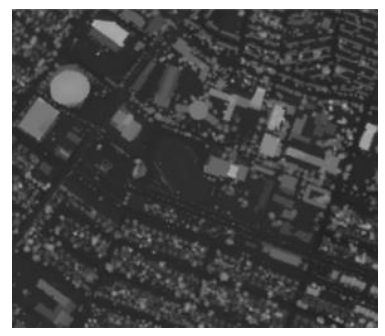

(a)

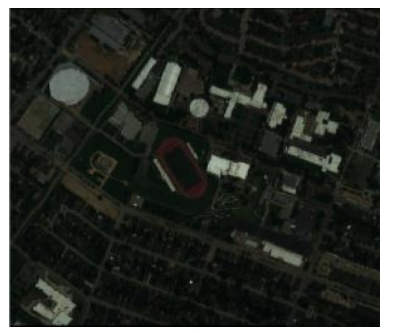

(b)
Figure 2. (a) LiDAR derived DSM (b) Hyperspectral imagery

Land cover classes consist of three types of grass (healthy, stressed and synthetic), road, soil, residential and commercial buildings. Spectral features (144 spectral bands, 30 vegetation indices and 3 PCs) have ability to discriminate different grass types and 2D objects; however referring to similar geometrical structure and height, LiDAR data cannot provide more information. On the other hand, spectral similarity of tree and grass/ roof and road may cause hyperspectral encounter some challenges but according to the height difference, fusion of hyperspectral imagery and LiDAR data may improve discrimination of complex urban objects.

\subsection{Feature Space Generation}

Generation of feature space is performed by processing both hyperspectral imagery and LiDAR data. Hyperspectral image was acquired by the CASI sensor and it has 144 bands. Moreover 30 vegetation indices are computed (Table 1). PCA transformation is applied on hyperspectral imagery and 3 first PCs with more than $99 \%$ eigenvalues are selected to complete spectral feature space. Consequently, the spectral feature space compose of 177 descriptors.

DSM derived from LiDAR data is a source of structural information. Geodesic morphological operation with circular structural element is applied on the DSM to create nDSM. Texture analysis of nDSM is performed based on GLCM features that 16 descriptors are extracted. Then roughness map and its 16 textural descriptors are also computed. Slope is further descriptor which is useful in classification, extracted from nDSM. Therefore the structural feature space is generated by merging all these 35 features.

By merging, spectral and structural feature space, a hybrid image is generated that contains rich information content for each pixel and forms our feature space with 212 features for pixel-based classification.

\subsection{Classification Based on SVM}

SVM classifier is applied to evaluate the quality of hybrid feature space. The SVM classification was done by using the LIBSVM through its Matlab interface (Chang and Lin, 2001). The Kappa coefficient and the overall accuracy are commonly used to determine the classification accuracy. These criteria were used to 
compare classification results and were computed by using the confusion matrix.

Ground truth samples are divided into training and testing data sets. The SVM classifier is trained based on training data and the best parameters are tuned and the classification performance is evaluated by unseen data (testing data). Among 7 classes, the classes tree, residential and commercial are placed in the "3D objects group", where fusion of LiDAR and hyperspectral data may improve classification results. For 2D objects, hyperspectral data are an efficient tool for discrimination among them. However 2D objects are commonly grouped as ground level in LiDAR data but the data are also useful in separating $2 \mathrm{D}$ and $3 \mathrm{D}$ objects.

Table 2 present the results of SVM classification along with determined parameters (based on grid search) for hyperspectral, LiDAR, spectral, spatial and hybrid feature space.

Table 2. Classification accuracy and SVM parameters

\begin{tabular}{lcccc}
\hline \multicolumn{1}{c}{ Dataset } & C & Gamma & Kappa & OA \\
\hline Hyperspectral & 128 & 1 & 0.82 & $84.78 \%$ \\
\hline LiDAR & 1028 & 8 & 0.29 & $33.65 \%$ \\
\hline Spectral & 64 & 0.25 & 0.84 & $86.74 \%$ \\
\hline Structural & 64 & 0.25 & 0.47 & $52.35 \%$ \\
\hline Hybrid Feature & 4 & 0.25 & 0.87 & $89.13 \%$ \\
\hline
\end{tabular}

Obtained results show that LiDAR data are not accurate enough to classify the dataset, however by extracting the structural features, the classification accuracy improves significantly. On the other side hyperspectral data show comparable results with respect to the hybrid feature space. However the hybrid image still exhibits a superior performance through the fusion of two datasets with different information content.

\subsection{Results of the Proposed Method}

Although the hybrid image improve the classification accuracy, but there are several correlated and redundant features which degrade classification performance. On the other side the SVM parameters is another important elements in classification. SVM parameters influence on feature subset selection and vice versa, therefore in this section simultaneous SVM parameters tuning and feature subset selection based on Bees Algorithm is performed. Table 3 contains important values for the Bees Algorithm.

Table 3. Parameters values of Bees Algorithm

\begin{tabular}{lc}
\hline \multicolumn{1}{c}{ Parameters } & Values \\
\hline Number of bee $(n)$ & 30 \\
\hline Number of best bees $(m)$ & 15 \\
\hline Number of elite $(e)$ & 5 \\
\hline Neighbourhood $\left(N_{e}\right)$ & 4 \\
\hline Neighbourhood best $\left(N_{m-e}\right)$ & 2 \\
\hline Iteration $(t)$ & 100 \\
\hline
\end{tabular}

Figure 3 shows the convergence plots for the Bees Algorithm procedures in spectral and structural features and hybrid feature space. The fitness value for the best individual in each generation is shown. The weight parameter in objective function (Equation 5) is set to $\rho=0.8$ which considers $80 \%$ of fitness to accuracy and $20 \%$ to dimensionality of feature space.

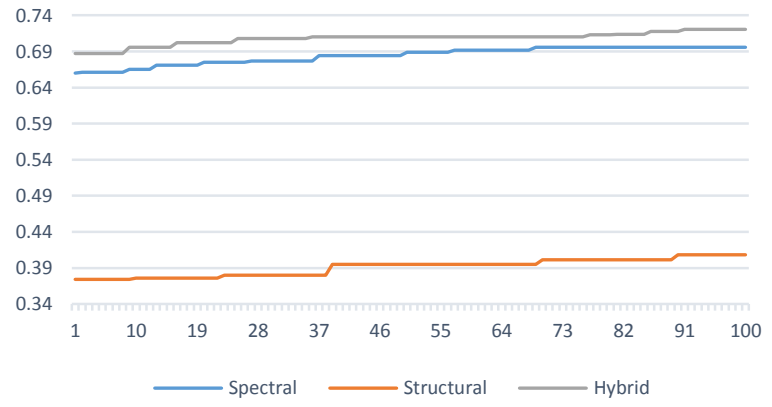

Figure 3. Convergence plot of the fitness value

Table 4 contains the number of selected features, as well as the values of regularization and kernel parameters and the classification accuracy for testing dataset, determined with the proposed method for spectral and structural and hybrid feature space.

Table 4. Results of the proposed method

\begin{tabular}{lccccc}
\hline \multicolumn{1}{c}{ Dataset } & \# Feature & C & Gamma & Kappa & OA \\
\hline Spectral & 81 & 131.5 & 0.175 & 0.87 & $89.65 \%$ \\
\hline Structural & 20 & 57.3 & 0.265 & 0.51 & $53.89 \%$ \\
\hline Hybrid & 101 & 76.34 & 0.274 & 0.901 & $92.53 \%$ \\
\hline
\end{tabular}

Analysing Table 4 reveals that applying the proposed method on hybrid image yields the best performance with respect to each dataset separately.

Comparing the results of hyperspectral imagery classification with the optimized classification system of hybrid images show that using DSM beside hyperspectral imagery and optimization of the SVM parameter and selection of feature subset, improve classification system approximately $8 \%$, moreover it eliminate 111 redundant features.

\section{CONCLUSION}

This study investigates the framework for optimization of a hybrid classification system to fuse hyperspectral and LiDAR data based on Bees Algorithm. Experiments were carried out using CASI hyperspectral image data and a DSM derived from LiDAR data. Several spectral and structural features were extracted from hyperspectral and LiDAR data respectively. Although SVM is an appropriate classifier for this high dimensional space, its performance is optimized by simultaneously determination of parameters and selection of feature subsets.

The obtained results show that utilizing 3D information from LiDAR data in addition to high spectral information of hyperspectral data, improves the classification performance. Optimization of the hybrid classification system based on Bees Algorithm improves classification accuracy about 3.5\% along with the elimination of 111 redundant features. Therefore the optimum hybrid classification system reaches more accurate results in a less complex space.

\section{ACKNOWLEDGEMENTS}

The authors would like to thank the Hyperspectral Image Analysis group and the NSF Funded Center for Airborne Laser 
Mapping (NCALM) at the University of Houston for providing the data sets used in this study, and the IEEE GRSS Data Fusion Technical Committee for organizing the 2013 Data Fusion Contest.

\section{REFERENCES}

Alonzo, M., Bookhagen, B., Roberts, D.A., 2014. Urban tree species mapping using hyperspectral and lidar data fusion. Remote Sensing of Environment, Vol. 148, pp. 70-83.

Brook, A., Ben-Dor, E., Richter, R., 2010. Fusion of Hyperspectra Images and LiDAR Data for Civil Engineering Structure Monitoring. $2^{\text {nd }}$ workshop on hyperspectral image and signal processing: Evolution in Remote Sensing (WHISPERS), pp. 1-5.

Chang, C. I. Hyperspectral Data Processing: Algorithm Design and Analysis, JohnWiley \& Sons, Inc., USA, 2013.

Dalponte, M., Bruzzone, L., and Gianelle, D., 2008. Fusion of Hyperspectral and LIDAR Remote Sensing Data for Classification of Complex Forest Area. IEEE Transaction on Geoscience and Remote Sensing, Vol. 46, No. 5, pp. 1416-1427.

Debes, C., Merentitis, A., Heremans, R., Hahn, J., Frangiadakis, N., van Kasteren, T., Liao, W., Bellens, R., Pizurica, A., Gautama, S. and Philips, W., Prasad, S., Du, Q., Pacifici, F., 2014. Hyperspectral and LiDAR data fusion: Outcome of the 2013 GRSS Data Fusion Contest. IEEE Journal of Selected Topics in Applied Earth Observations and Remote Sensing.

Fauvel, M., (2007). Spectral and Spatial Method for Classification of Urban Remote Sensing Data, Ph.D thesis.

Haralick, R.M., Shanmugam, K., Dinstein, I., 1973. Textural Features for Image Classification. IEEE Transaction on Systems, Man and Cybernetics, Vol. SMC-3, No. 6, pp. 610-621.

Hsu, C.W., Chang, C.C., and Lin, C.J., 2003. A Practical Guide to Support Vector Classification, National Technical Report, Taiwan University.

Khodadadzadeh, M., Li, J., Prasad, S., Plaza, A., (2015). Fusion of Hyperspectral and LiDAR Remote Sensing Data Using Multiple Feature Learning. IEEE Journal of Selected Topics in Applied Earth Observations and Remote Sensing, Vol. 8, No. 6.

Koetz, B., Morsdorf, F., van der Linden, S., Curt, T., Allgöwer, B., 2008. Multi-source Land Cover Classification for Forest Fire Management based on Imaging Spectrometry and LiDAR data. Forest Ecology and Management, Vol. 256, pp. 263-271.

Latifi, H., Fassnacht, F., Koch, B., 2012. Forest Structure Modeling with Combined Airborne Hyperspectral and LiDAR Data. Remote sensing of Environment, Vol. 121, pp.10-25.

Lin, S.W., Ying, K.C., Chen, S.C., and Lee, Z.J., 2008. Particle Swarm Optimization for Parameter Determination and Feature Selection of Support Vector Machines. Expert Systems with Applications, Vol. 35, No. 4, pp. 1817-1824.

Liu, Q.J., Jing, L.H., Wang, L.M., Lin, Q.Z., 2014. A Method of Particle Swarm Optimized SVM Hype-spectral Remote Sensing Image Classification. $35^{\text {th }}$ International Symposium on Remote Sensing of Environment.

Niemann, O.O., Gordon, W.F., Rafael, L., Fabio, V., 2009. LiDAR-Guided Analysis of Airborne Hyperspectral Data. First
Workshop on Hyperspectral Image and Signal Processing: Evolution in Remote Sensing, pp. 1-4.

O'Boyle, N.M., Palmer, D.S., Nigsch, F., and Mitchell, J.B.O., 2008. Simultaneous Feature Selection and Parameter Optimisation using an Artificial Ant Colony: Case Study of Melting Point Prediction. Chemistry Central Journal, Vol. 2, pp.21-25.

Ramdani, F., 2013. Urban Vegetation Mapping from Fused Hyperspectral Image and LiDAR Data with Application to Monitor Urban Tree Heights. Journal of Geographic Information System, 5(4).

Rashedi, E., Nezamabadi-pour, H., 2014. Feature Subset Selection Using Improved Binary Gravitational Search Algorithm. Journal of Intelligent and Fuzzy Systems, Vol. 26, No. 13, pp. 1211-1221.

Samadzadegan, F., Hasani, H., Schenk, T., 2012. Determination of optimum classifier and feature subset in hyperspectral images based on ant colony system. Photogrammetric Engineering \& Remote Sensing, Vol. 78, No. 12, pp. 1261-1273.

Sugumaran, R., Voss, M., 2007. Object-Oriented Classification of LiDAR-Fused Hyperspectral Imagery for Tree Species Identification in an Urban Environment. Urban Remote Sensing Joint Event, pp. 1-6.

Zhang, C., Qiu, F., 2012. Mapping Individual Tree Species in an Urban Forest using Airborne LiDAR Data and Hyperspectral Imagery, Photogrammetric Engineering and Remote Sensing. Vol. 78, No. 10, pp. 1079-1087. (AAG Remote Sensing Specialty Group 2011 Award Winner).

2013 IEEE GRSS Data Fusion Contest, Online: http://www.grssieee.org/community/technical-committees/data-fusion/ 